\title{
Weight-related hypothalamic dysfunction: a memorable case
}

\section{Osibogun OO, Okwerekwu FC, Oyibo SO}

Department of Endocrinology, Peterborough City Hospital, Peterborough, UK

The effect of weight loss on hypothalamic function is complex and not fully understood. There is interplay between neuropeptides (e.g., leptin, ghrelin) and the hypothalamus with the postulated aim of energy conservation and prevention of pregnancy during unfavourable conditions. We present a memorable case.

\section{CASE}

- A 35-yr-old lady presented with secondary amenorrhea of 17 years duration. She attained menarche at age 13. At age 18 her periods became scanty and then stopped. For this she had a 3-year course of the contraceptive pill to induce uterine withdrawal bleeding.

- She never exercised excessively but admits she did lose a stone in weight during examination stress as a teenager around the time her periods stopped.

- At presentation she had normal secondary sexual features and a body weight of $47.9 \mathrm{~kg}$ and height of $1.55 \mathrm{~m}$ (BMI 19.9).

\section{INVESTIGATION}

After stopping the contraceptive pill her endocrine profile revealed results consistent with hypogonadotrophic hypogonadism and mild central hypothyroidism (see baseline results in Table 1).

- Bone density scan revealed spinal osteopenia (Tscore -2.4) and MRI scan revealed a grossly normal pituitary gland.

\section{TREATMENT}

Because of ongoing tiredness, she had a trial of Levothyroxine $50 \mathrm{mcg}$ daily. Her TSH level fell to $0.02 \mathrm{mU} / \mathrm{L}$ while her FT4 rose above normal (23.8pmol/L). Upon advice we gradually withdrew the Levothyroxine. Her TSH rose to $0.1 \mathrm{mU} / \mathrm{L}$ and $\mathrm{FT} 4$ fell to $18.0 \mathrm{pmol} / \mathrm{L}$ on $25 \mathrm{mcg} /$ day of Levothyroxine, with further improvement (TSH $0.47 \mathrm{mU} / \mathrm{L}$, FT4 9.6pmol/L) six weeks after stopping Levothyroxine.

- She declined oestrogen replacement because of side-effects but continued calcium-vitamin D supplements.

Table 1 - Results of serial her endocrine profile and weight measurements (2014-2018)

\section{Blood test}

Oestradiol

Follicle stimulating hormone

Luteinising hormone

Prolactin

Testosterone

Thyroid Stimulating Hormone

Free Thyroxine

Free Triiodothyronine

Insulin Like Growth Factor

0 -min Pre-Synacthen Cortisol

30-minute Post-Synacthen Cortisol

Body Mass Index

$\begin{array}{cc}\begin{array}{c}\text { Normal reference } \\ \text { range }\end{array} & \begin{array}{c}\text { Baseline } \\ \mathbf{2 0 1 4}\end{array} \\ & \\ 100-750 \mathrm{pmol} / \mathrm{L} & <37 \\ 2-13 \mathrm{U} / \mathrm{L} & 5 \\ 1-13 \mathrm{U} / \mathrm{L} & 2 \\ 0-500 \mathrm{mU} / \mathrm{L} & 249 \\ 0.2-3.0 \mathrm{nmol} / \mathrm{L} & <0.2 \\ 0.3-4.2 \mathrm{mU} / \mathrm{L} & 1.35 \\ 12.0-22.0 \mathrm{pmol} / \mathrm{L} & 10.1 \\ 3.1-6.8 \mathrm{pmol} / \mathrm{L} & 3.1 \\ 16.1-39.8 \mathrm{nmol} / \mathrm{L} & 18.1 \\ - & 553 \\ >450 \mathrm{nmol} / \mathrm{L} & 876 \\ 18.5-24.9 & 19.9\end{array}$

\begin{tabular}{c|cc} 
& Follow-up results \\
$\mathbf{2 0 1 5}$ & $\mathbf{2 0 1 7}$ & $\mathbf{2 0 1 8}$ \\
& & \\
165 & 150 & 96 \\
7 & 6 & 10 \\
2 & 7 & 6 \\
162 & - & 233 \\
- & - & - \\
1.34 & 1.66 & 1.74 \\
10.3 & 10.5 & 10.2 \\
3.3 & 3.4 & 3.3 \\
- & - & - \\
- & - & - \\
- & - & - \\
21.4 & 21.2 & 21.0
\end{tabular}

\section{OUTCOME AND FOLLOW-UP}

Weight-loss-related hypothalamic dysfunction was discussed (particularly during her teenage years). Her weight did increase slightly along with her oestrogen levels. She remains well with a current weight of $50.4 \mathrm{~kg}$ and her thyroid function tests continue to show slightly low but stable FT4 levels.

\section{CONCLUSIONS}

This case highlights the complex interplay between weight loss and hypothalamic function. The resultant endocrine abnormalities, especially thyroid function and gonadal function, could be protective mechanisms and may revert to normal with weight gain. Hasty hormone replacement therapy could make things worse.

BES Glasgow - November 2018 